\title{
ON THE VARIETY OF NETS OF QUADRICS DEFINING TWISTED CUBICS
}

by

Geir Ellingsrud, Ragni Piene and Stein Arild Strømme

\section{§. INTRODUCTION}

Fix an algebraically closed field $k$ of characteristic 0 , and let $\mathrm{V}$ be a vector space over $\mathrm{k}$ of dimension 4 . Set $\mathbb{P}^{3}=\mathbb{P}(\mathrm{V})$, so that $V=\Gamma\left(\mathbb{P}^{3}, \Theta_{\mathbb{P}^{3}}(1)\right)$ and $s_{2}(V)=\Gamma\left(\mathbb{P}^{3}, \theta_{\mathbb{P}^{3}}(2)\right)$.

The main objective of this paper is the study of the variety $\mathrm{X} \subset \operatorname{Grass}_{3}\left(\mathrm{~S}_{2}(\mathrm{~V})\right)$ consisting of the nets of quadrics generated by the 2-minors of $(3 \times 2)$-matrices with linear forms as entries. The interest in $X$ stems from the fact that the space of twisted cubic curves may be considered as an open subset of $x_{i}$ in fact, any twisted cubic curve is defined by the vanishing of the 2-minors of a matrix as above.

We shall prove that $\mathrm{X}$ is smooth and compact. Hence it gives a natural compactification of the space of twisted cubics. Moreover, it will follow from the construction that $x$ is a minimal compactification in the sense that the complement in $\mathrm{X}$ of the space of twisted cubics is an irreduc.ible divisor. Furthermore, we compute - at least in principle - the Chow ring of $\mathrm{x}$ by giving algebra generators and relations.

Another compactification of the space of twisted cubics is the Hilbert scheme, or more precisely, the component $\mathrm{H}$ of $\mathrm{Hilb}^{3 \mathrm{~m}+1}\left(\mathbb{P}^{3}\right)$ containing the points corresponding to twisted cubics. In [P-S] it is shown that $\mathrm{H}$ is smooth. Furthermore, the complement in $\mathrm{H}$ of 
the space of twisted cubics is the union of $\mathrm{H}_{\mathrm{C}}$ and $\mathrm{H}_{\mathrm{e}^{\prime}}$ where $\mathrm{H}_{\mathrm{C}}$ consists of points corresponding to degenerate twisted cubics that are arithmetically Cohen-Macaulay, and $\mathrm{H}_{\mathrm{e}}$ to those that are not, i.e, that consist of a plane, singular cubic curve with an embedded point at a singular point. It is easy to see that all these degenerate curves are contained in exactly three linearly independent quadrics (for the Cohen-Macaulay curves this follows e.g. from [E], for curves with an embedded point this is shown in [P-S]). Hence there is a map $\mathrm{f}: \mathrm{H} \rightarrow \mathrm{X} \subset \operatorname{Grass}_{3}\left(\mathrm{~S}_{2}(\mathrm{~V})\right)$ which sends a curve to the net of quadrics containing it. Outside $\mathrm{H}_{e^{\prime}} f$ is an isomorphism, because a curve $\mathrm{C} \in \mathrm{H}-\mathrm{H}_{\mathrm{e}}$ is the intersection of the quadrics in $f(C)$.

If $C \in \mathrm{H}_{\mathrm{e}^{\prime}}$ then $\mathrm{C}$ is a plane cubic with an embedded point, and $f(C)$ is the net generated by $L_{0}^{2}, L_{0} L_{1}, L_{0} L_{2}$, where $L_{0}=0$ is an equation of the plane, and $L_{0}=L_{1}=L_{2}=0$ are equations of the point. Hence $f\left(\mathrm{H}_{e}\right)$ is isomorphic to the point-plane incidence correspondence I (which is embedded in $\operatorname{Grass}_{3}\left(\mathrm{~S}_{2}(\mathrm{~V})\right.$ ) as indicated above).

We strongly believe that $\mathrm{f}: \mathrm{H} \rightarrow \mathrm{X}$ is the blow-up of $\mathrm{X}$ along $I$. If this is true, we can compute the Chow ring of $\therefore$. We hope to report on this later.

The restriction of $f$ to $f^{-1}(I)_{\text {red }}=\mathrm{H}_{e} \rightarrow I$ is isomorphic to a $\operatorname{map} \mathbb{P}(N) \rightarrow I$, for some rank 7 bundle $N$ on $I$. Let $g: \tilde{\Pi} \rightarrow I$ denote the universal plane. Then $N$ is the subbundle of $g_{\star} \underset{\Pi}{\mathbb{\Pi}}(3)$ 
with fiber at $(P, \Pi)$ consisting of the cubics in $\Pi$ that are singular at $P$.

Knowing the Betti numbers of $X$ this suffices to compute the Betti numbers of $\mathrm{H}$ (see also $[\mathrm{Sch}]$ ).

Finally, we remark that because the natural action of a maximal torus in $\mathrm{PGL}(\mathrm{V})$ on $\mathrm{X}$ and on $\mathrm{H}$ has isolated fixed points, the Chow groups are equal to the homology groups, and they are all free abelian $[\mathrm{B} 1, \mathrm{~B} 2]$. 


\section{§2. THE CONSTRUCTION OF $\mathrm{X}$}

The points of the compactification $X$ of the space of twisted cubics are nets of quadrics that can be generated by the 2-minors of a (3×2)-matrix with linear forms as entries. We shall now make this connection explicit by exhibiting $x$ as a quotient space.

Let $E$ and $F$ be vector spaces of dimensions 3 and 2 respectively. Set $W=\operatorname{Hom}_{k}(F, E \otimes V)$. After a choice of bases for $E$ and $F$ we may consider an element $A \in W$ as a matrix ( $\left.a_{i j}\right)$, with $1 \leqslant i \leqslant 3,1 \leqslant j \leqslant 2$, with entries linear forms, $i . e ., a_{i j} \in V$.

For any matrix representation $\left(a_{i j}\right)$ of $A \in W$ the maximal minors generate the same subspace $E_{A}$ of $S_{2}(V)$. An intrinsic way of constructing $\mathrm{E}_{\mathrm{A}}$ is as follows: The map $\mathrm{A}$ induces a map $\mathrm{E}^{\vee} \rightarrow$ $F^{\vee} \otimes V$, hence a map $\Lambda^{2} E^{\vee} \rightarrow \Lambda^{2}\left(F^{\vee} \otimes V\right)$. Now there is a canonical map

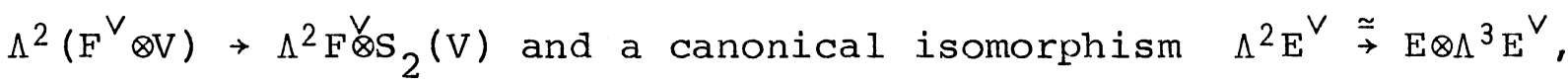
hence - after identifying the two 1-dimensional vector spaces $\Lambda^{3} E^{\vee}$ and $\Lambda^{2} F^{\vee}$ - we obtain a map $\lambda_{A}: E \rightarrow S_{2}(V)$, whose image is $E_{A}$. Note that $\lambda_{A}$ is uniquely defined up to a scalar, due to the choice of isomorphism $\Lambda^{3} \mathrm{E}^{\vee} \simeq \Lambda^{2} \mathrm{~F}^{\vee}$.

The group $G_{1}=G L(E) \times G L(F)$ acts on $W$ by $(g, h) A=g \otimes i d_{V} \cdot A \cdot h^{-1}$. Clearly the subgroup $\Gamma=\left\{\left(\alpha \cdot i d_{E}, \alpha \cdot i d_{F}\right): \alpha \in k^{\star}\right\}$ acts trivially on $W$, hence the group $G=G_{1} / \Gamma$ acts on $W$.

For technical reasons we shall consider $P=\mathbb{P}(W)$ and the action of $S=S L(E) \times S L(F)$ on $P$ induced by the action of $G$ on $W$. If $A \in W$, let $\bar{A} \in P$ denote the corresponding element. Denote by $\mathrm{U} \subset \mathrm{W}$ the set of maps $\mathrm{A}$ such that $\operatorname{dim} \mathrm{E}_{\mathrm{A}}=3$, and denote by $\overline{\mathrm{U}} \subset \mathrm{P}$ the image of $\mathrm{U}$. 
There is a map $\Psi: U \rightarrow \operatorname{Grass}_{3}\left(\mathrm{~S}_{2}(\mathrm{~V})\right)$ which sends $A$ to the net $\mathrm{E}_{\mathrm{A}} \cdot$ Clearly $\Psi$ factors through $\mathrm{X}$ and is G-invariant.

Proposition 1: There exists a projective, smooth geometric quotient $\bar{U} / S$ of $\bar{U}$ by $S$. The map $\bar{U} / S \rightarrow X$ induced by $\Psi$ is an isomorphism and $U \rightarrow X$ is a principal homogeneous bundle under $G$. The rest of this section is devoted to the proof of the above proposition.

Lemma 1: The following statements are equivalent.

(i) $\bar{A}$ is a semistable point under the action of $S$.

(ii) $\bar{A}$ is a stable point under the action of $S$.

(iii) $\operatorname{dim} \mathrm{E}_{\mathrm{A}}=3$.

Proof: Assume $\bar{A} \in P$ is not stable. Then there exists an element $(g, h) \in S$ and a 1-parameter subgroup $\lambda$ of $S$, on standard form, such that $\mu_{\lambda}\left(g \otimes i d_{V} \cdot \bar{A} \cdot h^{-1}\right) \leqslant 0$ (see $[N]$, Prop. 4.11). That $\lambda$ is on standard form means that

$$
\lambda(t)=\left(\left(\begin{array}{lll}
t^{\alpha} & 0 & 0 \\
0 & t^{\alpha_{2}} & 0 \\
0 & 0 & t^{\alpha_{3}}
\end{array}\right),\left(\begin{array}{ll}
t^{-\beta_{1}} & 0 \\
0 & t^{-\beta_{2}}
\end{array}\right)\right),
$$

where $\alpha_{1} \geqslant \alpha_{2} \geqslant \alpha_{3}, \beta_{1} \geqslant \beta_{2}$, and $\alpha_{1}+\alpha_{2}+\alpha_{3}=\beta_{1}+\beta_{2}=0$.

Clearly $\lambda(t) \cdot A=\left(t^{\alpha_{i}+\beta} j a_{i j}\right)$. If $\alpha_{i}+\beta_{j}<0$, we have $a_{i j}=0$, because $\bar{A}$ is not stable. Hence $a_{32}=0$ because $\alpha_{3}+\beta_{2}<0$. Suppose $\alpha_{2}+\beta_{2} \geqslant 0$ and $\alpha_{3}+\beta_{1} \geqslant 0$. Then, adding the two inequalities, we get $-\alpha_{1}=\alpha_{2}+\alpha_{3}+\beta_{1}+\beta_{2} \geqslant 0$, which contradicts the fact that $\alpha_{1}$ is the largest of three nonzero numbers whose sum is zero. Hence either $a_{22}=0$ or $a_{31}=0$, so $A$ is equivalent to a matrix of one of the following types: 


$$
\left(\begin{array}{ll}
\star & \star \\
\star & \star \\
0 & 0
\end{array}\right),\left(\begin{array}{ll}
\star & \star \\
\star & 0 \\
\star & 0
\end{array}\right) \text {. }
$$

On the other hand, it is easy to see that matrices of the above types give points of $P$ that are not semistable; in fact, we can use a 1-parameter subgroup with $\alpha_{1}=3, \alpha_{2}=2, \alpha_{3}=-5, \beta_{1}=1$, $\beta_{2}=-1$, in the first case, and one with $\alpha_{1}=5, \alpha_{2}=-2, \alpha_{3}=-3$, $\beta_{1}=4, \beta_{2}=-4$, in the second case. The following lemma then finishes the proof of Lemma 1 .

Lemma 2: Let $A \in W$. Then $\operatorname{dimE}_{A} \leqslant 2$ if and only if $A$ is equivalent under $G$ to a matrix of one of the above types, i.e., with $a_{32}=a_{31}=0$ or with $a_{32}=a_{22}=0$.

Proof: The maximal minors of such matrices are clearly not independent. Hence we may assume that dim $E_{A} \leqslant 2$. By performing row operations on $A$, we may assume that $A=\left(a_{i j}\right)$, with $a_{21} a_{32}=a_{31} a_{22}$. If $a_{32}=0$, then either $a_{31}=0$ or $a_{22}=0$, and we are done. If $a_{21}=0$, then either $a_{31}=0$ or $a_{22}=0$, and we are done by interchanging the two columns or the last two rows. If all four $a_{i j}$ 's are nonzero, we can write $a_{21}=\gamma a_{31}$ and $a_{32}=\gamma^{-1} a_{22}$, or $\mathrm{a}_{21}=\gamma \mathrm{a}_{22}$ and $\mathrm{a}_{32}=\gamma^{-1} \mathrm{a}_{31}$, with $\gamma \in \mathrm{k}^{\star}$; in both cases an obvious row operation puts $A$ on the desired form.

We conclude from Lemma 1 and the theory of $[M]$ (see $[N]$, Thm.3.14) that there exists a projective geometric quotient $\overline{\mathrm{U}} / \mathrm{S}$.

Lemma 3: Let $A \in W$ and let $J_{A} \subset C_{P}$ denote the sheaf of ideals generated by the quadrics of $E_{A}$. Assume $\operatorname{dim} E_{A}=3$ and that 
$V\left(J_{A}\right)$ is not a curve. Then $A$ can be represented by a matrix on the form

$$
\left(\begin{array}{cc}
0 & -L_{0} \\
L_{0} & 0 \\
-L_{1} & L_{2}
\end{array}\right),
$$

where $L_{0}, L_{1}, L_{2} \in V$ are linearly' independent.

Proof: If $V\left(J_{A}\right)$ is not a curve, then the 2-minors of $A$ have a common factor which is not a quadric, since otherwise $\operatorname{dim} \mathrm{E}_{\mathrm{A}} \leqslant 1$. Hence they must have a common linear factor. Because $V\left(J_{A}\right)$ has no component of codimension greater than 2 , the minors are $L_{0}^{2}, L_{0} L_{1}, L_{0} L_{2}$, for some linearly independent forms $L_{0}, L_{1}, L_{2}$. Since the relations between these minors obviously are the same as the relations between $L_{2}, L_{1}, L_{0}$, the columns of $A$ are linear combinations of the columns of the Koszul matrix

$$
K=\left(\begin{array}{ccc}
0 & \mathrm{~L}_{0} & -\mathrm{L}_{1} \\
-\mathrm{L}_{0} & 0 & \mathrm{~L}_{2} \\
\mathrm{~L}_{1} & -\mathrm{L}_{2} & 0
\end{array}\right)
$$

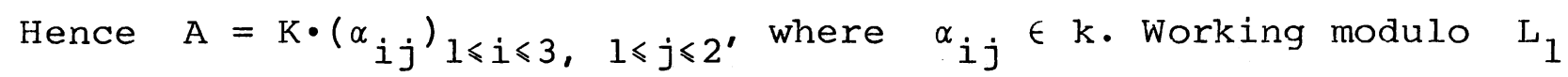
and $L_{2}$ we see that $\operatorname{det}\left(\alpha_{i j}\right)_{1 \leqslant i \leqslant 2,1 \leqslant j \leqslant 2} \neq 0$, and modulo $L_{0}$ we obtain $\alpha_{31}=\alpha_{32}=0$.

The map $\bar{U} / S \rightarrow X$, induced by $\Psi$, is bijective on closed points. In fact, on points corresponding to nets defining curves, this is clear because of the following. If a net $E_{A}$ defines a curve, this curve is (a possible degeneration of) a twisted cubic, which in turn determines the matrix $A$ up to the action by $G$. If $E_{A}$ does not define a curve, it defines by Lemma 3 a point-plane, and all matrices A defining this point-plane are equivalent under $G$, again by Lemma 3 . 
Lemma 4: For any point $A \in U$, the derivative $d_{A} \Psi$ of $\Psi$ at $A$ has rank 12 .

Before proving this lemma we observe that this finishes the proof of Proposition 1. In fact, since the map $\bar{U} / S \rightarrow X$ is bijective, the map $U \rightarrow X$ has connected fibers. Hence, by Lemma 4, $X$ is smooth, and thus $\overline{\mathrm{U}} / \mathrm{S} \rightarrow \mathrm{X}$ is an isomorphism because of Zariski's Main Theorem. Now it is easy to see that $\bar{U} / S$ is a quotient of $U$ by $G\left(U \rightarrow \bar{U}\right.$ is a $k^{\star}$-bundle, and $G$ is an extension of $k^{\star}$ by $\left.S\right)$, so we may identify $U / G=\bar{U} / S=X$. To show that $U \rightarrow X$ is a principal homogeneous bundle under $G$, it therefore suffices ( $[M], 0.9)$ to check that $G$ acts freely on $U$. There are two cases to consider. Assume first that $A \in U$ is such that $V\left(M_{A}\right)$ is a curve. Then there is a resolution of $\mathrm{J} A$ on $\mathbb{P}^{3}$,

$$
0 \rightarrow 2(+3) \stackrel{A}{\rightarrow} 3 \theta(-2) \rightarrow Y_{A} \rightarrow 0
$$

Assume $(g, h) \in G_{1}$ stabilizes $A$. Then, since $\underline{\operatorname{Hom}}_{\mathrm{P}^{3}}\left(\mathrm{~J}_{A^{\prime}} J_{A}\right)=k^{*}$, we have

$$
\begin{aligned}
& 0 \rightarrow 2 C^{\prime}(-3) \stackrel{\mathrm{A}}{\rightarrow} 3 \mathrm{C}^{+}(-2) \rightarrow \mathrm{J}_{\mathrm{A}} \rightarrow 0 \\
& i \downarrow h \quad l \downarrow g \quad \downarrow \alpha \\
& 0 \rightarrow 2 \mathrm{O}(-3) \stackrel{\mathrm{A}}{\rightarrow} 3 C_{+}(-2) \rightarrow \mathrm{J}_{\mathrm{A}} \rightarrow 0 \text {. }
\end{aligned}
$$

Then $g-\alpha \cdot i d$ induces a map $3(-2) \rightarrow 2 f(-3)$, which must be zero - hence $g=\alpha \cdot i d$. This implies $h=\alpha \cdot i d$, hence $(g, h) \in \Gamma$, i.e., A has trivial stabilizer in $G$. In the case $V\left(Y_{A}\right)$ is not a curve, by Lemma 3, A can be represented by a matrix on the form

$$
\left(\begin{array}{cc}
0 & -\mathrm{L}_{0} \\
\mathrm{~L}_{0} & 0 \\
-\mathrm{L}_{1} & \mathrm{~L}_{2}
\end{array}\right),
$$


and one verifies by direct computation that such an A has trivial stabilizer in $\mathrm{G}$.

Proof of Lemma 4: Since $\operatorname{dim} X=12$ and $\operatorname{dim} U=24, \operatorname{rank} \mathrm{d}_{\mathrm{A}} \Psi=12$ for general points $A \in U$. There are obvious actions on $U$ and on $\operatorname{Grass}_{3}\left(S_{2}(V)\right)$ by PGL(V), under which $\Psi$ is equivariant. Hence the set of points $A \in U$ where rank $d_{A} \Psi<12$ is invariant under the action of PGL(V), and - if nonempty - contains at least one closed orbit. The only closed orbits in $U$ are the orbit consisisting of matrices defining point-planes and that of matrices defining the full second order neighborhood of a line. Therefore we may assume that $A$ is one of the following matrices

$$
A_{1}=\left(\begin{array}{cc}
0 & -x_{0} \\
x_{0} & 0 \\
-x_{1} & x_{2}
\end{array}\right), \quad A_{2}=\left(\begin{array}{cc}
x_{0} & 0 \\
x_{1} & x_{0} \\
0 & x_{1}
\end{array}\right) .
$$

The tangent space to $U$ at $A$ is just $W$, and that of $\operatorname{Grass}_{3}\left(S_{2}(V)\right)$ at $\Psi(A)$ is $\operatorname{Hom}_{k}\left(E_{A^{\prime}} S_{2}(V) / E_{A}\right)$. A tangent vector $\tau$ to $U$ at $A$ is given as $\tau=A+\varepsilon L$, where $L \in W$ and $\varepsilon^{2}=0$. The map $d_{A} \Psi(\tau)$ sends a minor of $A$ to the $\varepsilon$-part of the corresponding minor of $\tau$. Hence $d_{A} \Psi(\tau)=0$ is equivalent to the three relations

(*) $\quad\left|\begin{array}{ll}a_{i 1} & l_{i 2} \\ a_{j 1} & l_{j 2}\end{array}\right|+\left|\begin{array}{ll}l_{i 1} & a_{i 2} \\ l_{j 1} & a_{j 2}\end{array}\right| \in E_{A}$

for $1 \leqslant i<j \leqslant 3$.

Now Lie $\left(G_{1}\right)=\operatorname{Lie}(G L(E)) \oplus \operatorname{Lie}(G L(F))$ acts on $\operatorname{Ker}\left(d_{A} \Psi\right)$ via

$$
\left(i d_{E}+\varepsilon B\right)(A+\varepsilon L)\left(i d_{F}+\varepsilon C\right)=A+\varepsilon(L+B A+A C)
$$


where $B \in$ Lie(GL(E)) and $C \in$ Lie(GL(F)). It will be enough to show that $\operatorname{Ker}\left(d_{A} \Psi\right)$ is the orbit of $A+\varepsilon \cdot 0$, because $\operatorname{dim} \operatorname{Ker}\left(d_{A} \Psi\right) \geqslant 12$ and the orbit is of dimension $\leqslant 12$ since Lie $(\Gamma)=\left\{\left(\gamma \cdot i d_{E^{\prime}}-\gamma \cdot i d_{R}\right): \gamma \in k\right\}$ acts trivially.

We may replace $\tau \in \operatorname{Ker}\left(d_{A} \Psi\right)$ by any other element in the orbit of $\tau$. Hence, if $A=A_{1}$, we may assume

$$
\tau=\left(\begin{array}{cc}
0 & -x_{0} \\
x_{0} & 0 \\
-x_{1} & x_{2}
\end{array}\right)+\varepsilon\left(\begin{array}{ll}
l_{11} & \ell_{12} \\
l_{21} & \ell_{22} \\
l_{31} & l_{32}
\end{array}\right)
$$

with $l_{11}, l_{21}, l_{31} \in \mathrm{k}\left[\mathrm{x}_{2}, \mathrm{x}_{3}\right]$. Using the relations

(*) with $i=2, j=3$ and $i=1, j=3$, we see that $\ell_{11}=l_{21}=0$, and that all $l_{i j} \in k\left[x_{0}, x_{1}, x_{2}\right]$. It is now easy to produce an element $(B, C) \in \operatorname{Lie}\left(G_{1}\right)$ such that $\left(l_{i j}\right)=B A_{1}+A_{1} C$.

If $\mathrm{A}_{\mathrm{A}} \mathrm{A}_{2}$, then we may assume

$$
\tau=\left(\begin{array}{cc}
x_{0} & 0 \\
x_{1} & x_{0} \\
0 & x_{1}
\end{array}\right)+\varepsilon\left(\begin{array}{ll}
\ell_{11} & \ell_{12} \\
\ell_{21} & \ell_{22} \\
\ell_{31} & l_{32}
\end{array}\right),
$$

with $l_{12}, l_{22}, l_{32} \in \mathrm{k}\left[\mathrm{x}_{2}, \mathrm{x}_{3}\right]$. Using the relations $(\star)$ with $i=2$, $j=2$ and $i=1, j=3$, we see that $l_{12}=l_{32}=0$ and that all $l_{i j} \in \mathrm{k}\left[\mathrm{x}_{0}, \mathrm{x}_{1}\right]$, so that $\ell_{22}=0$. As above we can then write $\left(l_{i j}\right)=B_{2}+A_{2} C$ for some $(B, C) \in \operatorname{Lie}\left(G_{1}\right)$. 
§3. THE CHOW RING OF $\mathrm{X}$

We start by constructing two bundles on $x, \mathcal{E}$ and $\mathcal{F}$, of ranks 3 and 2 respectively, whose Chern classes are algebra generators for the Chow ring $A(X)$ of $x$. The idea is to try to descend the bundles $E_{U}$ and $F_{U}$ on $U$ to $X$. clearly $G_{1}$ acts on $E_{U}$ and $F_{U}$ as follows. If $(g, h) \in G_{1}$ and $(e, u) \in E_{U}=E \times U$, then $(g, h)(e, u)$ $=\left(\right.$ ge, guh ${ }^{-1}$ ), and similarly on $F_{U}$. Since $\Gamma \subset G_{1}$ does not act trivially on these bundles, we do not get an induced action by $\mathrm{G}=$ $G_{1} / \Gamma$. However, if $\lambda=\operatorname{Hom}_{k}\left(\Lambda^{3} E, \Lambda^{2} F\right), i . e ., \lambda$ corresponds to the character $(g, h) \rightarrow \frac{\operatorname{det} h}{\operatorname{det} g}$, it is easily seen that $\Gamma$ acts trivially on the $\mathrm{G}_{1}$-bundles $\mathrm{E}_{\mathrm{U}}^{\prime}=\mathrm{E}_{\mathrm{U}} \otimes_{\mathrm{k}} \lambda$ and $\mathrm{F}_{\mathrm{U}}^{\prime}=\mathrm{F}_{\mathrm{U}} \otimes_{\mathrm{k}} \lambda$. Hence $\mathrm{E}_{\mathrm{U}}^{\prime}$ and $\mathrm{F}_{\mathrm{U}}^{\prime}$ are G-bundles, and because $\mathrm{G}$ acts freely on $\mathrm{U}$, these bundles descend to bundles $\mathcal{E}$ and $F$ on $x$.

Since by definition $W=\operatorname{Hom}_{k}(F, E \otimes V)$, there is a universal map $\tilde{A}: \quad F_{U} \rightarrow E_{U} \otimes V$ on $U$, and hence also a map $\tilde{A} \otimes i d_{\lambda}: F_{U}^{\prime} \rightarrow E_{U}^{\prime} \otimes V$. This map is G-equivariant and descends to a map $\alpha: \mathcal{F} \rightarrow \mathcal{E} \otimes \mathrm{V}$ on $\mathrm{X}$.

Proposition 2: The Chern classes of $\mathcal{E}$ and $\mathcal{F}$ generate the Chow ring $A(X)$ as a $\mathbb{Z}$-algebra.

Proof: The group $G_{1}$ is a structure group for the bundle $\mathcal{E} \oplus \tilde{F}$, so we may construct the principal $G_{1}$-homogeneous bundle $\Phi: T \rightarrow X$ associated with $\xi \oplus F$. Two things should be observed. Firstly, the Chow ring of $\mathrm{T}$ is, via $\Phi^{\star}$, isomorphic to $A(X)$ modulo the ideal generated by the Chern classes of $\mathcal{E}$ and $\mathcal{F}$ ([C], Rernarques, p. 435). Secondly, since $\Phi^{\star} \mathcal{E}$ and $\Phi^{\star} \mathcal{F}$ are trivial, $\Phi$ factors through $\Psi: U \rightarrow X$, and it is easily seen that the induced map $\mathrm{T} \rightarrow \mathrm{U}$ is a 
$\mathrm{k}^{\star}$-bundle. Therefore the Chow rings of $\mathrm{U}$ and $\mathrm{T}$ are isomorphic. Now $U$ is an open subset of the affine space $W$, and so $A(U)=\mathbf{Z}$. This proves the proposition.

Remark that $c_{1}(\tilde{E})=c_{1}(\tilde{F})$; in fact, as G-bundles, $\Lambda^{3} \mathrm{E}_{\mathrm{U}}^{\prime} \cong \Lambda^{2} \mathrm{~F}_{\mathrm{U}^{\prime}}^{\prime}$ hence we have $\Lambda^{3} \mathcal{E} \cong \Lambda^{2} F$. Furthermore, by the definition of $\Psi$, the restriction to $x$ of the universal subbundle $Q$ of $\mathrm{s}_{2}(\mathrm{~V})$ on $\operatorname{Grass}_{3}\left(\mathrm{~S}_{2}(\mathrm{~V})\right)$ is $\mathcal{E}$.

Let $\pi: Y=\operatorname{Grass}_{2}(Q \otimes V) \rightarrow \operatorname{Grass}_{3}\left(\mathrm{~S}_{2}(\mathrm{~V})\right)$ denote the Grassmann bundle of rank 2 subbundles of $Q \otimes V$, and let $R$ denote the universal subbundle of $Q \otimes V$ on $Y$. The map $\alpha: \mathcal{F} \rightarrow \xi \otimes V$ induces an embedding $i: X \rightarrow Y$. In fact, $\alpha$ gives $\mathcal{F}$ as a subbundle of $\mathcal{V} \otimes \mathrm{V}$ because if $\alpha$ is not injective at a point represented by a $(3 \times 2)-$ matrix $A$, the two columns of $A$ are linearly dependent, hence all the 2-minors vanish. This is impossible, so we get a map $i: X \rightarrow Y$, which - being a section over $x$ of the projection $\mathrm{Y} \rightarrow \operatorname{Grass}_{3}\left(\mathrm{~S}_{2}(\mathrm{~V})\right)$ - is an embedding.

Proposition 3: The class of $X$ in $A(Y)$ is given by

$$
[x]=\frac{1}{m}\left[c\left(\pi^{\star} Q^{\vee}\right)^{10}\left(1+c_{1}(R)-c_{1}\left(\pi^{\star} Q\right)\right)^{-1}\right]_{29^{\prime}}
$$

where $\mathrm{m}$ is some positive integer.

Proof: On $\mathrm{Y}$ there are two inclusions, $\pi^{\star} \mathrm{Q} \rightarrow \mathrm{S}_{2}(\mathrm{~V})_{\mathrm{Y}}$ and $\pi^{\star}\left(Q \otimes \Lambda^{3} Q^{-1}\right) \otimes \Lambda^{2} R \rightarrow S_{2}(V)_{Y^{\prime}}$ the latter being constructed from $\Re \rightarrow \pi^{\star} Q \otimes V$ in the same way as we constructed $\lambda_{A}$ in $\S 2$. The points of $X$ correspond to nets of quadrics that are generated by the 2-minors of a $(3 \times 2)$-matrix, so it is clear that $x$ consists 
of the points of $Y$ where the two above maps of bundles are proportional. Hence $\mathrm{X}$ is set-theoretically the scheme $\mathrm{Z}$ defined by the $2-$ minors of the map

$$
\mathcal{V}_{Y} \oplus\left(\Lambda^{2} R \otimes \Lambda^{3} \pi^{\star} Q^{-1}\right) \rightarrow \underline{\operatorname{Hom}}_{Y}\left(\pi^{\star} Q, S_{2}(V)\right) .
$$

Since $X$ has the "right" codimension 29, the class of $Z$ is given by Porteous' formula as $[\mathrm{Z}]=\left[\mathrm{c}\left(\pi^{\star} Q^{\vee}\right)^{10}\left(1+c_{1}(\Omega)-c_{1}\left(\pi^{\star} Q\right)\right)^{-1}\right]_{29}$. Since $\mathrm{X}$ is irreducible, $[\mathrm{Z}]=\mathrm{m}[\mathrm{X}]$ for some positive integer $\mathrm{m}$. This finishes the proof. (Probably $z$ is reduced, so that $m=1$ holds, but we don't need this.)

Theorem 1: The Chow ring of $X$ is given by $A(X)=A(Y) / O$, where

$$
\pi=\operatorname{Ann}\left(\left[c\left(\pi^{\star} Q_{2}^{V}\right)^{10}\left(1+c_{1}(\Omega)-c_{1}\left(\pi^{\star} Q\right)\right)^{-1}\right]_{29}\right) .
$$

Proof. Recall that $\mathcal{E}=i^{\star} \pi^{\star} \mathcal{Q}$ and $\mathcal{F}=i^{\star} \mathcal{R}$, where $i: X \rightarrow Y$ is the embedding. Since $A(Y)$ is generated by the Chern classes of $\pi^{\star} Q$ and $\mathcal{R}$, and $A(X)$ by those of $\mathcal{E}$ and $\mathcal{F}$ (by Proposition 2), the $\operatorname{map} i^{\star}: A(Y) \rightarrow A(X)$ is surjective, hence $([S], \ldots)$, $\operatorname{Ker}\left(i^{\star}\right)=\operatorname{Ann}([\mathrm{x}])$. The theorem then follows from Proposition 3 and the fact that $A(Y)$ is a free abelian group.

As a byproduct of the fact that the Chern classes of $\mathcal{E}$, and $F$ generate $A(X)$ (Proposition 2) and the knowledge of the topological Euler-Poincaré characteristic $e(x)$ of $x$, we obtain the Betti numbers of $\mathrm{X}$ : 


\begin{tabular}{c|ccccccc}
$\mathrm{i}$ & 0 & 1 & 2 & 3 & 4 & 5 & 6 \\
\hline $\mathrm{b}_{2 i}=\mathrm{b}_{2(12-i)}$ & 1 & 1 & 3 & 4 & 7 & 8 & 10
\end{tabular}

To see this, we use that $e(x)=58$ (this will be shown below). Let $\mathrm{R}=\oplus \mathrm{R}_{\mathrm{i}}$ denote the free, graded $\mathrm{z}$-algebra with one generator in degree 1 , two in degree 2 , and one in degree 3 . Then $A(X)$ is a quotient of $R$ by a graded ideal $J=\oplus_{i}$. The dimensions of the $R_{i}$ are:

\begin{tabular}{c|rrrrrrr}
$i$ & 0 & 1 & 2 & 3 & 4 & 5 & 6 \\
\hline $\operatorname{dim} R_{i}$ & 1 & 1 & 3 & 4 & 7 & 9 & 14
\end{tabular}

Then $2 \sum_{i=0}^{5} \operatorname{dim} R_{i}+\operatorname{dim} R_{6}=64$. Hence, if $x=\sum_{i=0}^{5} \operatorname{dim} J_{i}$ and $y=\operatorname{dim} J_{6}$, we get $2 x+y=6$. Clearly $x=0, y=6$ is impossible, since $b_{10} \leqslant b_{12}$. Furthermore, $y=0$ is impossible because if $J_{i} \neq 0$ for some $i<6$, then $J_{6} \neq 0$. Assume $x=y=2$. If $J_{4} \neq 0$, then $\operatorname{dim} J_{6} \geqslant 3$, so $J_{4}=0$ and $\operatorname{dimJ}_{5}=2$. It follows that $J_{6}=t J_{5}$, where $t$ is the generator of degree 1 . The locally split bundle map on $X$, $\mathcal{E}^{\vee} \rightarrow \mathcal{F}^{\vee} \otimes v$, gives a relation of degree 6 between the Chern classes of $\varepsilon$ and $\mathcal{F}$, namely $\left[\mathrm{c}\left(\xi^{\vee}\right) c\left(\mathcal{E}^{\vee}\right)^{-1}\right]_{6}=0$. This gives an element of $J_{6}$ which is not a multiple of $t$. So the only possibility left is $\mathrm{x}=1, \mathrm{y}=4$, and we are done.

The Euler-Poincaré characteristic of $\mathrm{X}$ is computed using the action of a maximal torus of $P G L(V)$ on $X$. The fixed points are isolated and finite in number, and their number equals $e(X)$ $[B 1, B 2]$. If a fixed point of $X$ corresponds to a curve, the support of this curve is contained in the tetrahedron of reference. Hence the curve is either three non coplanar edges (there are 16 such), one edge doubled in a plane (face) union a consecutive edge not contained in that plane (there are 24 such), or the full second 
order neighborhood of one edge (there are 6 of these). Since the fixed points that do not correspond to curves lie in $I$, there are $e(I)=12$ of these. Adding up gives $e(X)=58$.

Using the fact that the map $\mathrm{f}: \mathrm{H} \rightarrow \mathrm{X}$ gives an isomorphism $\mathrm{H}-\mathrm{H}_{e} \stackrel{\sim}{\rightarrow} \mathrm{X}-\mathrm{I}$ and that the restriction of $f$ to $\mathrm{H}_{e}$ is a bundle $P(N) \rightarrow I$, we obtain the Betti numbers of $\mathrm{H}$.

\begin{tabular}{c|rrrrrrr}
$i$ & 0 & 1 & 2 & 3 & 4 & 5 & 6 \\
\hline$b_{2 i}=b_{2(12-i)}$ & 1 & 2 & 6 & 10 & 16 & 19 & 22
\end{tabular}

Up to now we have studied determinantal nets of quadrics in $\mathbb{P}^{3}=\mathbb{P}(V)$. However, our methods are independent of the dimension of $\mathrm{V}$, and all the proofs carry over to the general case dimV $=n+1$, with the obvious modifications. Hence we have

Theorem 2: Let $V$ be a vector space of dimension $n+1$. Let $\mathrm{X}_{\mathrm{n}} \subset \operatorname{Grass}_{3}\left(\mathrm{~S}_{2}(\mathrm{~V})\right)$ denote the space of determinantal nets of quadrics in $P(V)$. Then $X_{n}$ is a smooth, projective variety, and its Chow ring is given by

$$
A\left(X_{n}\right)=A\left(Y_{n}\right) / \sigma_{n}
$$

where $Y_{n}=\operatorname{Grass}_{2}(Q \otimes V)$ and

$$
q_{n}=\operatorname{Ann}\left(\left[c\left(\pi^{\star} Q^{\vee}\right)^{(n+2)}\left(1+c_{1}(R)-c_{1}\left(\pi^{\star} Q\right)\right)^{-1}\right]_{3\left(\begin{array}{c}
n+1 \\
2
\end{array}\right)-1}\right) \text {. }
$$




\section{$\underline{\text { References }}$}

[B1] A. Bialynicki-Birula, "Some theorems on actions of algebraic groups", Ann. of Math., 98 (1973), 480-497.

[B2] -, "Some properties of the decompositions of algebraic varieties determined by actions of a torus", Bull.Acad.(-)Pol.Sc., Série Sc.math. astr.phys., 24(1976), 667-674.

[C] Séminaire Chevalley, Anneaux de Chow et applications, Paris 1958.

[E] G. Ellingsrud, "Sur le schéma de Hilbert des variétés de codimension 2 dans $\mathrm{P}$ a cone de Cohen-Macaulay", Ann.Scient.Ec.Norm.Sup., $8(1975), 423-432$.

[M] D. Mumford, J. Fogarty, Geometric Invariant Theory, 2nd Enlarged Ed., Ergebnisse der Math. 34, Springer-Verlag 1982.

[N] P.E. Newstead, Introduction to moduli problems and orbit spaces, Tata Institute, Springer-Verlag, Bombay 1978.

[P-S] R. Piene, M. Schlessinger, "On the Hilbert scheme compactifiaction of the space of twisted cubics", Amer.(-) J.Math., 107 $(1985), 761-774$.

[Sch] D. Schaub, "Sur 1'homologie du schéma de Hilbert des cubiques de genre arithmétique nul", C.R.Acad.Sc.Paris, t.301, Série I $(1985), 307-310$.

[S] S.A. Str $\phi$ mme, "On parametrized rational curves in Grassmann varieties", Proceedings of the conference in Rocca di Papa on Projective Space Curves, june 1985.

G. Ellingsrud, R. Piene

Universitetet $i$ Oslo

Matematisk institutt

P.B. 1053 Blindern

$\mathrm{N}-0316$ Oslo 3

NORWAY
S.A. Str $\phi$ mme

Universitetet $i$ Bergen

Matematisk institutt

Allégt. 55

IV-5014 Bergen

NORWAY 